\title{
On the Charged Bose Liquid
}

\author{
A.A. Shanenko \\ Bogoliubov Laboratory of Theoretical Physics \\ Joint Institute for Nuclear Research \\ 141980, Dubna, Moscow region, Russia
}

\begin{abstract}
A new approach developed in Ref.1 for investigating the spatial boson correlations at low temperatures is considered in the particular case of the Coulomb interaction potential. This approach is based on integro-differential equations for the radial distribution function and can be used beyond the weak coupling regime.
\end{abstract}

\section{PACS: 05.30.Jp, 67.90.+z}

Description of the charged Bose liquid, or, in other words, of the strongly correlated many-boson system with the Coulomb interparticle potential, is a long-standing problem of the condensed matter physics. Recently, interest in this problem has been renewed in the connection with the noteworthy results of applying the Bose and Bose-Fermi liquid models [2] to the explanation of various phenomena inherent in the superconductors with high critical temperatures. These models are based on the Bogoliubov approach [3] implying the weak coupling regime. For the system of charged bosons the weak coupling approximation can be used at small values of the Brueckner parameter $r_{S} \sim 0.1$. However, questions requiring detailed investigations often concern the materials with relatively low densities of charge carriers which correspond to $r_{S}$-values even larger than those of the electron gas at 
the metallic densities. For example, there are the results [4] testifying to the possibility of forming the so-called large bipolaron (e.g. the two-electron cluster with a large radius of electron-electron separation, see papers in Ref. [5]) inside the polaron environment at the electron densities $\sim 10^{19} \mathrm{~cm}^{-3}$ close to the carrier densities of the heavily doped degenerate polar semiconductors. To investigate the possible phase transition of the charge carrier system from the polaron state to the bipolaron phase at these densities, one should operate with the charged Bose liquid of large bipolarons for $r_{S} \gg 1$. Indeed, the "large-bipolaron " Bohr radius can be expressed [4] as

$$
a_{b i p}=\frac{\hbar^{2}}{m_{b i p} Q_{b i p}^{2}} \quad\left(Q_{b i p}=\frac{2 e}{\varepsilon_{0}^{1 / 2}}\right)
$$

where $\varepsilon_{0}$ is the static dielectric constant of the polar material considered, $m_{\text {bip }}$ denotes the bipolaron mass, and $e$ is the electron charge. To connect $a_{b i p}$ with the canonical electron Bohr radius $a_{e}=\frac{\hbar^{2}}{m_{e} e^{2}}=5.29 \cdot 10^{-9} \mathrm{~cm}$, we use the reasonable estimate 汭 $m_{\text {bip }} \approx 2 m_{\text {pol }}$ $\left(m_{p o l}\right.$ is the polaron mass) and the relation $m_{p o l} \sim \frac{16 \alpha^{4}}{81 \pi^{2}} m_{e}^{*} \quad\left(m_{e}^{*}\right.$ is the band mass) correct at the values of the Fröhlich electron-phonon coupling constant $\alpha \sim 7$ typical for the large bipolaron [5]. This leads to $a_{b i p} \sim 0.0026 \varepsilon_{0} \frac{\hbar^{2}}{m_{e}^{*} e^{2}}$. Further, with the tables of the physical properties of polar materials [6] we find that $\varepsilon_{0} \sim 10 \div 100$ and $m_{e}^{*} \sim 0.1 \div 0.5 m_{e}$ represent the most frequent case. Choosing, say, $\varepsilon_{0}=100$ and $m_{e}^{*}=0.1 m_{e}$, we obtain $a_{b i p} \sim 2.6 a_{e}=1.375 \cdot 10^{-8} \mathrm{~cm}$. With this choice the "large-bipolaron "Brueckner parameter $r_{S}=\frac{1}{a_{b i p}}\left(\frac{3}{4 \pi n}\right)^{1 / 3}$ has values $\sim 20$ at the bipolaron densities $n \sim 10^{19} \mathrm{~cm}^{-3}$, which proves the statement mentioned above. Note that another choice gives even larger values of $r_{S}$. Thus, to make the final conclusions on the possibility of the phase transition from the Fermi state to the Bose one in the charge carrier systems of various materials and to clear up the status of the Bose liquid models [2], one should deal with the Bose many-particle systems beyond the weak coupling regime.

The aim of this letter is to consider potentialities of a new approach to investigating the spatial correlations of cold bosons in the particular case of the Coulomb interaction potential. The approach is based on integro-differential equations for the function of the 
radial boson distribution that have been derived in paper [1] in a way analogous to that of Ref. [7]. For the charged bosons the simplest of these equations is written as

$$
\frac{\hbar^{2}}{m g^{1 / 2}(r)} \triangle g^{1 / 2}(r)=\frac{Q^{2}}{r}+n \int \frac{Q^{2}}{|\vec{r}-\vec{y}|}(g(y)-1) d \vec{y} .
$$

where $g(r)$ denotes the radial distribution function, $m$ and $Q$ are the boson mass and charge, $n$ is the particle density. Besides, $\triangle$ stands for the Laplacian. To be convinced of the capacity of the equation (四) for giving reasonable estimates of the radial distribution function of the charged bosons, let us consider the weak coupling regime characterized by

$$
g(r)=1-\varepsilon(r), \quad \varepsilon(r) \ll 1 .
$$

Using (22) and only keeping the terms linear in $\varepsilon(r)$, we arrive at the linear version of the equation (11):

$$
-\frac{\hbar^{2}}{2 m} \triangle \varepsilon(r)=\frac{Q^{2}}{r}-n \int \frac{Q^{2}}{|\vec{r}-\vec{y}|} \varepsilon(y) d \vec{y}
$$

which can easily be solved via the Fourier transformation. The Fourier transform of $\varepsilon(r)$ obeys the relation

$$
\frac{\hbar^{2} q^{2}}{2 m} \widetilde{\varepsilon}(q)=\frac{4 \pi Q^{2}}{q^{2}}-n \frac{4 \pi Q^{2}}{q^{2}} \widetilde{\varepsilon}(q) .
$$

Introducing the new variable $x \equiv q / A$ with $A^{4}=16 \pi n m Q^{2} / \hbar^{2}$, from (4) we find

$$
\widetilde{\varepsilon}(q)=\frac{f_{\text {est }}(x)}{n}, \quad f_{\text {est }}(x)=1-\frac{x^{4}}{x^{4}+0.5} .
$$

It is now interesting to compare the estimate (5) with the result [8] of the random phase approximation (RPA)

$$
\widetilde{\varepsilon}(q)=\frac{f_{R P A}(x)}{n}, \quad f_{R P A}(x)=1-\frac{x^{2}}{\sqrt{x^{4}+1}} .
$$

Short-range correlations are determined by the behaviour of $\widetilde{\varepsilon}(q)$ at $x=q / A \gg 1$ for which we have

$$
f_{\text {est }}(x) \simeq \frac{1}{2 x^{4}}, \quad f_{R P A}(x) \simeq \frac{1}{2 x^{4}} \quad(x \gg 1) .
$$


There is also good agreement between $f_{\text {est }}(x)$ and $f_{R P A}(x)$ in the region $x \sim 1$ ruling the spatial correlations at the intermediate distances close to the screening radius $1 / A$ :

$$
\begin{gathered}
f_{\text {est }}(1.5)=0.090, \quad f_{R P A}(1.5)=0.086 ; \\
f_{\text {est }}(1)=0.333, \quad f_{R P A}(0.5)=0.293 ; \\
f_{\text {est }}(0.5)=0.889, \quad f_{R P A}(0.5)=0.758 ;
\end{gathered}
$$

When $x \ll 1$ (long-range correlations), we again obtain close numerical results

$$
f_{\text {est }}(0.2)=0.997, \quad f_{R P A}(0.2)=0.960
$$

however, $f_{\text {est }}(x)$ and $f_{R P A}(x)$ tend to unity in completely different ways:

$$
f_{\text {est }}(x) \simeq 1-2 x^{4}, f_{R P A}(x) \simeq 1-x^{2} \quad(x \ll 1) .
$$

The comparative consideration of $f_{\text {est }}(x)$ and $f_{R P A}(x)$ allows one to make the following two conclusions. The first, negative, is that the model presented is not able to yield the correct behaviour of the structure factor $S(k)=1-n \widetilde{\varepsilon}(k)$ at small $k$ and, therefore, the correct spectrum of the low-lying excitations given by $k^{2} / S(k)$ (see Ref. [9]). The second conclusion, positive, consists in that the equation (11) can provide a good estimate of the pair correlation function $h(r)=g(r)-1$ for distances $r \ll 1 / A$ and $r \sim 1 / A$ exactly where the spatial boson correlations are significant.

These conclusions have been found in the weak coupling regime. And what can we expect, as to the results of (1), beyond the weak coupling, for $r_{S}>1$. It is known [10] that RPA should be corrected for $r_{S}>1$ to avoid the trouble of negative values of $g(r)$ at small particle separations. This occurs because the short-range correlations are taken into account not in the best way within RPA. At $r_{S}$ small enough the short-range correlations do not yield any essential contribution to the thermodynamic quantities. Therefore, this shortcoming of RPA does not practically affect the results for $r_{S}<1$. On the contrary, at large $r_{S}$ the short-range correlations become significant and influence the thermodynamic quantities considerably. In this case RPA leads not only to the negative values of $g(r)$ at 
small $r$ but overestimates the absolute value of the ground state energy. As to the model based on the equation (11), it has no problem like this. Indeed, at small $r$ where

$$
\frac{Q^{2}}{r} \gg n \int \frac{Q^{2}}{|\vec{r}-\vec{y}|}(g(y)-1) d \vec{y},
$$

the expression (1) can be written as

$$
-\frac{\hbar^{2}}{m} \triangle g^{1 / 2}(r)+\frac{Q^{2}}{r} g^{1 / 2}(r)=0
$$

It is nothing else but the Schrödinger equation like those which are often used to investigate the spatial boson correlations at small particle separations as well as in dilute systems [1]. Thus, we may expect that the relation (11) will also give reasonable results on the charged boson correlations for $r_{S}>1$.

If we have a reliable estimate of $g(r)$, we can obtain adequate estimates of the thermodynamic quantities of the system considered. In particular, it is possible to calculate $E$, the ground state energy of the charged bosons in the neutralizing background, with the following well-known relation [11:

$$
\frac{E}{N}=\frac{E_{i d}}{N}+\frac{1}{2} n \int_{0}^{1} d \gamma \int(g(r, \gamma)-1) \frac{Q^{2}}{r} d \vec{r}
$$

Here $N$ denotes the number of bosons, $E_{i d}$ is the energy of noninteracting particles (in our case $\left.E_{i d}=0\right)$. The quantity $\gamma$ is the coupling constant and $g(r, \gamma)$ denotes the solution of the equation obtained with the replacement $Q^{2} \rightarrow \gamma Q^{2}$ in (1). On the basis of the relation $($ 可) we find

$$
g(r, \gamma)=1-\frac{1}{(2 \pi)^{3} n} \int\left(1-\frac{q^{4}}{q^{4}+\gamma 0.5 A^{4}}\right) \exp (\imath \vec{q} \vec{r}) d \vec{q}
$$

which can be rewritten as

$$
g(r, \gamma)=1-\frac{\gamma^{1 / 2} A^{2}}{2 \pi^{2} n r} \int_{0}^{\infty} y \sin \left(\gamma^{1 / 4} A r y\right)\left(1-\frac{y^{4}}{y^{4}+0.5}\right) d y
$$

Substituting (10) into (8) we obtain

$$
\frac{E}{N}=-\frac{Q^{2} A}{\pi} \int_{0}^{1} d \gamma \int_{0}^{\infty} d l \int_{0}^{\infty} d y \gamma^{1 / 4} y \sin (l y)\left(1-\frac{y^{4}}{y^{4}+0.5}\right)
$$


The result of numerical integration

$$
\int_{0}^{\infty} d l \int_{0}^{\infty} d y y \sin (l y)\left(1-\frac{y^{4}}{y^{4}+0.5}\right)=0.9339
$$

makes it possible to derive from (11) the relation

$$
\frac{E}{N}=-0.2378 Q^{2} A=-0.885 r_{S}^{-3 / 4} R y
$$

where $r_{S}$ and $R y$ obey the standard definitions

$$
r_{S}=\frac{m Q^{2}}{\hbar^{2}}\left(\frac{3}{4 \pi n}\right)^{1 / 3}, \quad R y=\frac{m Q^{4}}{2 \hbar^{2}}
$$

The RPA result for $E / N$ can easily be found with the relation (6). This yields the expression

$$
\frac{E}{N}=-\frac{Q^{2} A}{\pi} \int_{0}^{1} d \gamma \int_{0}^{\infty} d l \int_{0}^{\infty} d y \gamma^{1 / 4} y \sin (l y)\left(1-\frac{y^{2}}{\sqrt{y^{4}+1}}\right) .
$$

Numerical integration

$$
\int_{0}^{\infty} d l \int_{0}^{\infty} d y y \sin (l y)\left(1-\frac{y^{2}}{\sqrt{y^{4}+1}}\right)=0.8468
$$

allows one to obtain

$$
\frac{E}{N}=-0.2156 Q^{2} A=-0.803 r_{S}^{-3 / 4} R y
$$

Thus, agreement between the results of (12) and (14) is within the accuracy of $10 \%$. Besides, it is also interesting to make a remark on the next-to-leading contribution to the mean energy of the system. Within the approach considered this contribution can be calculated substituting

$$
g(r)=1-\varepsilon_{1}(r)-\varepsilon_{2}(r) \quad\left(\varepsilon_{2}(r) \ll \varepsilon_{1}(r) \ll 1\right)
$$

into the equation (1). Omitting details of the calculations, let us only write the final result

$$
\frac{E}{N}=\left(-0.885 r_{S}^{-3 / 4}+\text { const }\right) R y, \quad \text { const } \approx 0.08
$$

which can be compared with the relation 


$$
\frac{E}{N}=\left(-0.803 r_{S}^{-3 / 4}+0.212\right) R y
$$

found within the Bogoliubov scheme [3].

Summarizing, let us recall the basic points of the letter once more. The new approach of investigating the spatial correlations of cold bosons has been considered in the particular situation of a many-boson system with the Coulomb interaction potential. In this case the radial distribution function $g(r)$ can be estimated with the integro-differential equation (1D). As it has been shown, this equation is able to yield reasonable results on the short- and intermediate-range correlations. However, the obtained decay of the function $h(r)=g(r)-1$ at distances essentially larger than the screening radius is not correct. Nevertheless, Eq.(11) has provided a good estimate of the ground state energy at small $r_{S}$. This result gives an optimistic view on the possibility of using Eq.(1) to explore the thermodynamic behavior of the charged Bose liquid $\left(r_{S}>1\right)$ where the influence of the short-range correlations on the system properties is essentially more significant than that for $r_{S}<1$.

Acknowledgement: The author thanks A. Yu. Cherny for interest in the subject of this letter and stimulating discussions. 


\section{REFERENCES}

[1] A. A. Shanenko, Phys. Lett. A, 1997 (to be published), see cond-mat/9612195.

[2] R. Friedberg, T. D. Lee, Phys. Lett. A 138, 423 (1989); R. Micnas, J. Ranninger, and S. Robaszkiewicz, Rev. Mod. Phys. 62, 113 (1990); A. S. Alexandrov, JETP Lett. 55, 195 (1992); A. S. Alexandrov, N. F. Mott, Supercond. Sci. Technol. 6, 215 (1993); N. F. Mott, Physica C 205, 191 (1993); G. Iadonisi, V. Cataudella, D. Ninno, M. I. Chiofalo, Phys. Lett. A 196, 359 (1995).

[3] N. N. Bogoliubov, J. Phys. USSR 11, 23 (1947); L. L. Foldy, Phys. Rev. 124, 649 (1961); A. S. Alexandrov, W. H. Beere, Phys. Rev. B51, 5887 (1995).

[4] A. A. Shanenko, M.A Smondyrev, and J. T. Devreese, Solid State Comm. 98, 1091 (1996).

[5] J. Adamowski, Phys. Rev. B 39, 3649 (1989); G. Verbist, F. M. Peeters, and J. T. Devreese, Phys. Rev. B 43, 2712 (1991); F. Bassani, M. Geddo, G. Iadonisi, and D. Ninno, Phys. Rev. B 43, 5296 (1991); M. A. Smondyrev, J. T. Devreese, and F. M. Peeters, Phys. Rev. B 51, 15008 (1995).

[6] J. W. Hodby. In: 'Polarons in Ionic Crystals and Semiconductors', ed. J. T. Devreese (North-Holland, Amsterdam, 1972) p. 389; E. Kartheuser, ibid, p.717.

[7] A. A. Shanenko, Phys. Rev. E54, 4420 (1996).

[8] A. Isihara, M. Wadati, Phys. Rev. 183, 312 (1969).

[9] R. P. Feynman, Phys. Rev. 94, 262 (1954).

[10] K. S. Singwi, M. P. Tosi, R. H. Land, A. Sjölander, Phys. Rev. 176, 589 (1968); K. S. Singwi, A. Sjölander, M. P. Tosi, R. H. Land, Phys. Rev. B1, 1044 (1970); P. Vashishta, K. S. Singwi, Phys. Rev. B6, 875 (1972).

[11] Kittel C., Quantum Theory of Solids (Wiley, New York, 1963). 